\title{
Immunology and Diabetes Workshops: report of the first international workshop on the standardisation of cytoplasmic islet cell antibodies
}

\section{Summary of a workshop organised by the Juvenile Diabetes Foundation International held in Monte Carlo on 31 October and 1 November 1985}

\author{
Report prepared by: G. F. Bottazzo ${ }^{1}$ and H. Gleichmann ${ }^{2}$ (chairpersons) \\ ${ }^{1}$ The Middlesex Hospital, Department of Immunology, Arthur Stanley House, London, U. K. and \\ ${ }^{2}$ Diabetes Research Institute, University of Düsseldorf, Federal Republic of Germany
}

In the past decade, since islet cell antibodies (ICA) were reported in Type 1 (insulin-dependent) diabetes mellitus, this serological marker has been used increasingly in diabetes research. ICA determinations have been applied for various clinical purposes, including attempts to classify diabetes, monitor diabetic patients after diagnosis and identify susceptible individuals at risk of developing the disease. Recently, the significance of these autoantibodies in patients undergoing immunomodulating therapy, including islet and segmental pancreas transplantation, has been investigated. These approaches have indicated the potential of ICA determination to further investigate the disease process.

Following the first description of the assay, various modifications of the standard immunofluorescence method have been introduced. To date, however, no attempt has been made to standardise the test to enable inter-laboratory comparisons. For this reason, the Workshop was established. Twenty-six laboratories participated in the Workshop. Coded sera and a standard protocol were sent to each laboratory and the participants were asked to submit their results together with those obtained by various specified modifications. This report deals primarily with analysis of results obtained using the following protocol:
1. Use $4 \mu \mathrm{m}$ unfixed cryostat sections of Blood Group 0 pancreas, air-dried $15-30 \mathrm{~min}$ at room temperature.

2. Put test serum $(25 \mu 1)$ onto sections and incubate at room temperature for $20 \mathrm{~min}$ in a closed humid box.

3 . Wash off serum with PBS $(0.15 \mathrm{~mol} / 1, \mathrm{pH} 7.2)$ and leave slides standing in excess PBS for $15 \mathrm{~min}$.

4. Apply your routine FITC conjugate at appropriate dilution in PBS for $20 \mathrm{~min}$.

5. Repeat step 3 and mount slide under glass cover-slip using PBS/glycerol or similar mounting medium.

Determine end-point titres which are the reciprocal of the dilution. Neat serum is expressed as a titre of 1 ; a two-fold dilution, e. g. $25 \mu 1$ serum plus $25 \mu \mathrm{lBS}$, is expressed as a titre of 2 ; a fourfold dilution, e.g. $25 \mu \mathrm{l}$ serum plus $75 \mu \mathrm{l}$ PBS, is expressed as a titre of 4 , etc.

An analysis of the results of the various modifications will be published separately. The modifications investigated were acetone-fixation of sections, heat-inactivation of sera, complementfixing ICA, prolonged incubation with aprotinin, and the use of alternative conjugates such as FITC-Protein A, glucose-oxidase and counterstaining with monoclonal antibodies binding to islet cell determinants.

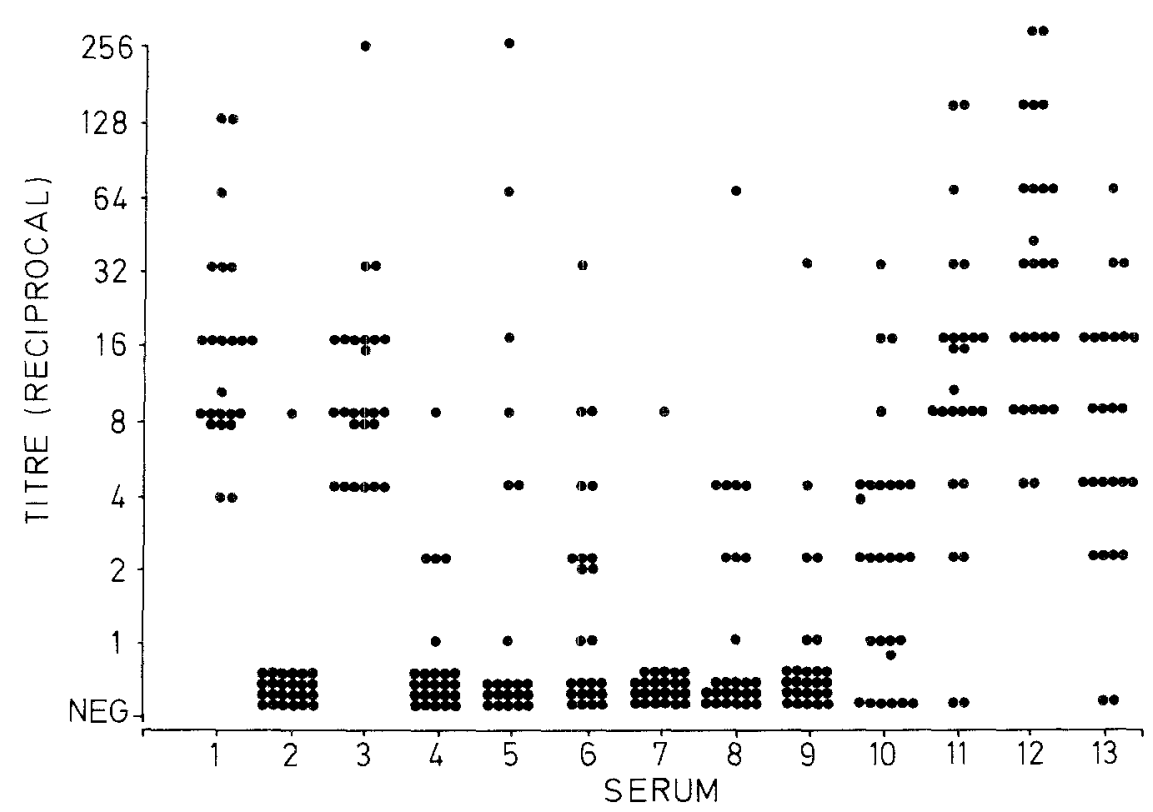

Fig. 1. End-point titres of ICA obtained by 24 laboratories with 13 coded sera 

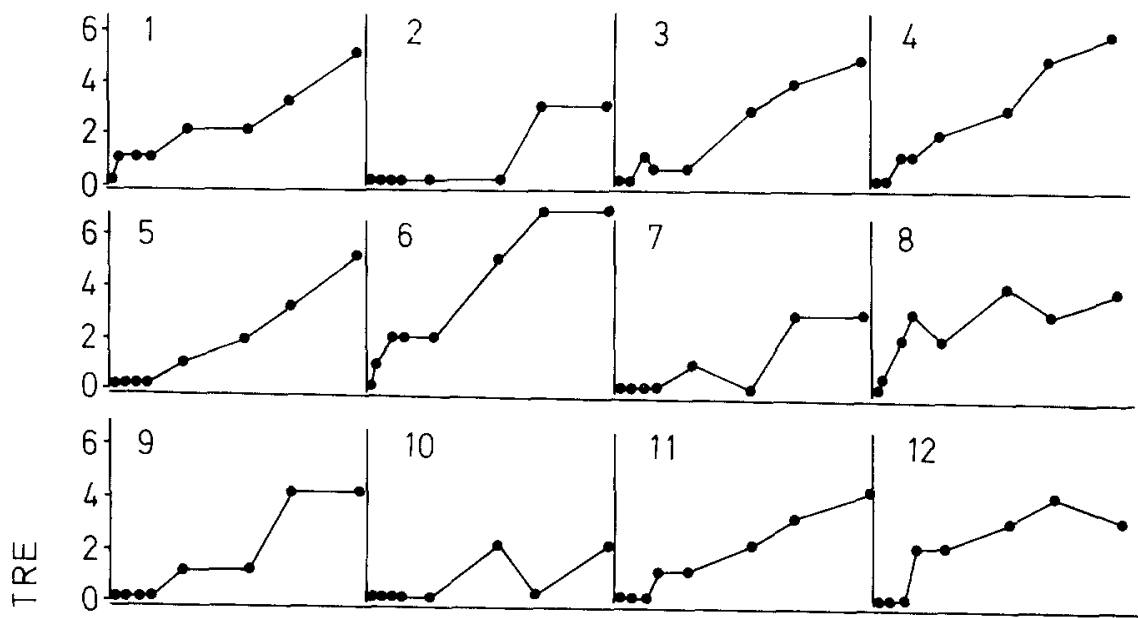

10
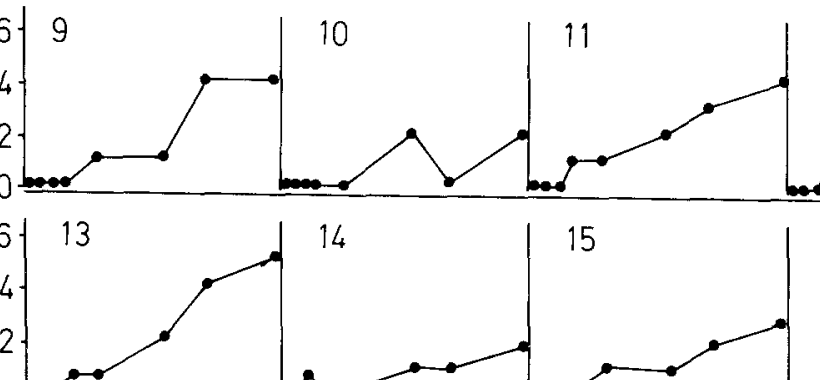

12
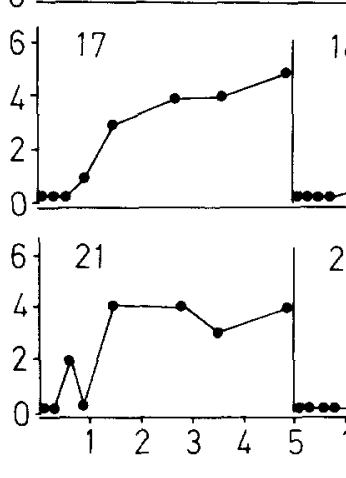

22

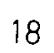

18
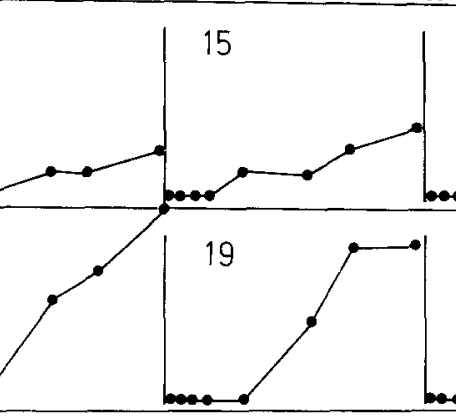

16

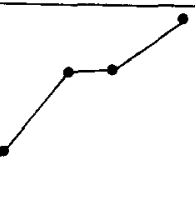

19
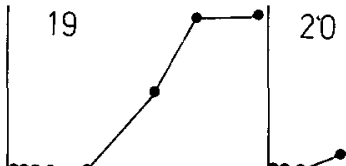

20

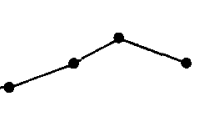

ASSIGNED VALUE (MEAN LOG 2 TITRE)

\section{Summary of results}

ICA end-point titres were determined in the coded sera by each participating laboratory (Fig. 1). Concordance for ICA was $90 \%$ for sera with high titres (Serum Nos. 1, 3, 11, 12,13) and sera reported as negative $(2,7)$. Sample 1 and 2 were disclosed to be positive and negative, respectively. Concordance for sera nos. 4, 5, 6, 8,9 and 10 ranged between $52-79 \%$. On the whole, these sera were recorded as negative or with low titres. The lower concordance may be attributable to factors such as variation of pancreatic substrates. The presence of interfering antibodies such as mitochondrial and nuclear present in serum 5 and 7 , respectively, might also have affected the results.

Experience with standardisation of an immunofluorescence test for anti-nuclear antibodies has revealed the value of standard curves for improving concordance, precision and accuracy. Accordingly, standard curves based on titres reported from 24 laboratories were computed for eight of the thirteen sera shown in Figure 1 by E. Bonifacio, Perth, Australia. These interim standards provided a range of values covering low to high ICA levels (Fig. 2). Each interim standard was assigned a value, i. e. the geometric mean of the corresponding end-point titres observed in all laboratories. Standard curves for the individual laboratories were obtained by plotting the assigned values (abcissa) against the corresponding observed titre ( $\log _{2}$, ordinate axis).

Linearly increasing standard curves are demonstrated for most laboratories. Results are in the expected order based on the assigned values, but inter-laboratory quantitative differences between sera are demonstrated. In 4 laboratories $(8,10,21,22)$ linear standard curves were not obtained, and in two others $(7,12)$ they were questionable. At present it cannot be concluded whether this preliminary analysis reflects poor precision or true variation from the expected values.

When observed titres for 2 sera not included in the standard curve were converted into units derived from the standard curve, the inter-laboratory variation decreased, illustrating that the use of standards and standard curves improves concordance. Thus, these preliminary results indicate that the availability of reference sera should allow standard curves to be established and used to define ICA in common units. The ultimate aim is to establish precision and accuracy so that inter-laboratory results may be compared with confidence.

\section{Proposed Stage 2 Workshop}

Reference sera are now available and can be requested by laboratories who wish to standardise their ICA test. This first exchange of sera will allow identification of standard sera for future reference work and will permit each laboratory to standardise its ICA assay.

Expression of results in common units will overcome the need for a standard method. Each laboratory can then assess the validity of its own method regardless of modifications introduced.

Requests for workshop sera by laboratories interested in participating in the Stage 2 Workshop and willing to submit their results for analysis should be addressed to: Helga Gleichmann, CoChairman and Secretary of the Immunology and Diabetes Workshops Committee.

Dr. Helga Gleichmann

Diabetes-Forschungsinstitut

Auf'm Hennekamp 65

D-4000 Düsseldorf 1

FRG 